\title{
Asymptotic formulas for coefficients of inverse theta functions
}

\author{
Kathrin Bringmann and Jan Manschot
}

\begin{abstract}
We determine asymptotic formulas for the coefficients of a natural class of negative index and negative weight Jacobi forms. These coefficients can be viewed as a refinement of the numbers $p_{k}(n)$ of partitions of $n$ into $k$ colors. Part of the motivation for this work is that they are equal to the Betti numbers of the Hilbert scheme of points on an algebraic surface $S$ and appear also as counts of Bogomolny-Prasad-Sommerfield (BPS) states in physics.
\end{abstract}

\section{Introduction and statement of results}

Jacobi forms were first systematically studied by Eichler and Zagier [14] and enjoy a wide variety of applications in the theory of modular forms, combinatorics $[37,38]$, conformal field theory $[12,24]$, black hole physics $[10$, 13], Hilbert schemes of points [18], Donaldson invariants [19] and many other topics. This paper focuses on a class of negative index Jacobi forms with a single order pole in the elliptic variable $w$. The analysis of the coefficients of such functions is more complicated then the well-understood class of Jacobi forms which depend holomorphically on $w$. It turns out that these Fourier coefficients (in $w$ ) are not modular but related to quantum modular forms $[15,40]$. The appearance of these functions in the above mentioned topics calls for an explicit knowledge of their coefficients and in particular of their asymptotic growth. In this paper, we provide such asymptotic formulas. One of the immediate motivations is the counting of Bogomolny-PrasadSommerfield (BPS) states in physics and in particular those with vanishing angular momentum. This motivation is explained in more detail after stating the results. 


\subsection{Statement of results}

We consider the following class of negative weight $1-k / 2(k \in \mathbb{N})$ and index $-1 / 2$ Jacobi forms

$$
h_{k}(w ; \tau):=\frac{i}{\theta_{1}(w ; \tau) \eta(\tau)^{k-3}}
$$

with $(q:=\exp (2 \pi i \tau), \zeta:=\exp (2 \pi i w))$

$$
\begin{aligned}
\theta_{1}(w ; \tau) & :=i \zeta^{\frac{1}{2}} q^{\frac{1}{8}} \prod_{n \geq 1}\left(1-q^{n}\right)\left(1-\zeta q^{n}\right)\left(1-\zeta^{-1} q^{n-1}\right) \\
\eta(\tau) & :=q^{\frac{1}{24}} \prod_{n \geq 1}\left(1-q^{n}\right) .
\end{aligned}
$$

We are interested in two expansions of $h_{k}(w ; \tau)$. The first expansion is in terms of the coefficients $a_{m, k}(n)$, defined by:

$$
q^{\frac{k}{24}}\left(\zeta^{\frac{1}{2}}-\zeta^{-\frac{1}{2}}\right) h_{k}(w ; \tau)=: \sum_{\substack{n \geq 0 \\ m \in \mathbb{Z}}} a_{m, k}(n) \zeta^{m} q^{n}, \quad|\zeta q|,\left|\zeta^{-1} q\right|<1
$$

Note that $\sum_{m \in \mathbb{Z}} a_{m, k}(n)=p_{k}(n)$, where $p_{k}(n)$ denotes the number of partitions of $n$ into $k$ colors. These are enumerated by $\eta(\tau)^{-k}$ :

$$
\sum_{n \geq 0} p_{k}(n) q^{n-\frac{k}{24}}=\frac{1}{\eta(\tau)^{k}}
$$

For $k=1$, Equation (1.1) corresponds to the generating function of the crank of partition [17], and for $k=2$ to the birank [20].

The second expansion is motivated from physics, and is based on the fact that the coefficients of $q$ are Laurent polynomials, symmetric under $\zeta \rightarrow \zeta^{-1}$ and with maximal degree $n$. Therefore, we can express $h_{k}(w ; \tau)$ as:

$$
q^{\frac{k}{24}}\left(\zeta^{\frac{1}{2}}-\zeta^{-\frac{1}{2}}\right) h_{k}(w ; \tau)=\sum_{m, n \geq 0} b_{m, k}(n) \chi_{2 m+1}\left(\zeta^{\frac{1}{2}}\right) q^{n}
$$

with

$$
\chi_{m}(\zeta):=\frac{\zeta^{m}-\zeta^{-m}}{\zeta-\zeta^{-1}}, \quad b_{m, k}(n):=a_{m, k}(n)-a_{m+1, k}(n)
$$


Following the approach of Wright [38], we determine all polynomial corrections to the leading exponential of the coefficients $a_{m, k}(n)$ in the large $n$ limit.

Theorem 1.1. We have for $N \geq 1$ :

$$
\begin{aligned}
a_{m, k}(n)= & (2 \pi)^{-\frac{k}{2}} \sum_{\ell=1}^{N} d_{m, k}(\ell) n^{-\frac{2+2 \ell+k}{4}}\left(\pi \sqrt{\frac{k}{6}}\right)^{1+\ell+\frac{k}{2}} \\
& \times I_{-1-\ell-\frac{k}{2}}\left(\pi \sqrt{\frac{2 k n}{3}}\right)+O\left(n^{-1-\frac{N}{2}-\frac{k}{4}} e^{\pi \sqrt{\frac{2 k n}{3}}}\right),
\end{aligned}
$$

where $d_{m, k}(\ell)$ are defined by Equation (2.2) and $I_{\ell}(x)$ is the usual I-Bessel function. Here the error term depends on $k$ and $m$.

Theorem 1.1 allows us to compare the asymptotic growths of $a_{m, k}(n)$ for different values of $m$. The asymptotic behavior of the Bessel function:

$$
I_{\ell}(x)=\frac{e^{x}}{\sqrt{2 \pi x}}\left(1+O\left(x^{-1}\right)\right)
$$

directly yields:

Corollary 1.2. We have

$$
a_{m, k}(n)-a_{r, k}(n)=\pi^{3}\left(r^{2}-m^{2}\right)(8 n)^{-\frac{9+k}{4}}\left(\frac{k}{3}\right)^{\frac{k+7}{4}} e^{\pi \sqrt{\frac{2 k n}{3}}}+O\left(n^{-3-\frac{k}{4}} e^{\pi \sqrt{\frac{2 k n}{3}}}\right),
$$

where the error term depends on $m, k$ and $r$.

From Theorem 1.1 the asymptotics of $b_{m, k}(n)$ for large $n$ immediately follow:

Corollary 1.3. We have

$$
b_{m, k}(n)=(2 m+1) \pi^{3}(8 n)^{-\frac{9+k}{4}}\left(\frac{k}{3}\right)^{\frac{k+7}{4}} e^{\pi \sqrt{\frac{2 k n}{3}}}+O\left(n^{-3-\frac{k}{4}} e^{\pi \sqrt{\frac{2 k n}{3}}}\right)
$$

where the error term depends on $m$ and $k$. 
Note that this corollary shows that $b_{m, k}(n)$ increases with $m$ in the limit of large $n$. Beyond the validity of equation $(1.3), b_{m, k}(n)$ eventually decreases with increasing $m$ for fixed $n$, and in particular $b_{m, k}(n)=0$ for $m>n$.

We next compare the asymptotic behavior of the coefficients $b_{0, k}(n)$ with those of $p_{k}(n)$. It is well known that the asymptotic growth of the latter is given by $[21,30]$ :

$$
p_{k}(n)=2\left(\frac{k}{3}\right)^{\frac{1+k}{4}}(8 n)^{-\frac{3+k}{4}} e^{\pi \sqrt{\frac{2 k n}{3}}}+O\left(n^{-\frac{5+k}{4}} e^{\pi \sqrt{\frac{2 k n}{3}}}\right) .
$$

Thus, we find for the ratio $b_{0, k}(n) / p_{k}(n)$ :

$$
\frac{b_{0, k}(n)}{p_{k}(n)}=\frac{\pi^{3}}{16}\left(\frac{k}{3 n}\right)^{\frac{3}{2}}+O\left(n^{-2}\right) .
$$

\subsection{Motivation: moduli spaces and BPS states}

BPS states of both gauge theory and gravity have been extensively studied in the past for a variety of reasons. These states are representations of the $\mathrm{SU}(2)_{\text {spin }}$ massive little group in four dimensions labeled by their angular momentum or highest weight $J$. The subset of BPS states with vanishing angular momentum $(J=0)$, also known as "pure Higgs states" [4], have recently attracted much interest $[4,25,28,34]$. The states with $J=0$ are in some sense more fundamental. In particular in gravity, these states are candidates for microstates of single center black holes, and as such are the states relevant for studies of the Bekenstein-Hawking area law of black hole entropy.

Within string theory it is possible to obtain exact generating functions of the degeneracies of classes of BPS states. The asymptotic growth as function of the angular momentum is for example previously studied in [7,9,33]. String theory relates BPS states to the cohomology of moduli spaces of sheaves supported on a Calabi-Yau manifold. From this perspective the $\mathrm{SU}(2)_{\text {spin }}$ representations correspond to representations of the Lefshetz SL(2) action on the cohomology of the moduli space $\mathcal{M}[36]$. The states with $J=0$ correspond to the part of the middle cohomology which is invariant under the Lefshetz action.

In the present work, we consider moduli spaces of semi-stable sheaves supported on a complex algebraic surface $S$, which can be thought of as being embedded inside a Calabi-Yau manifold. If $S$ is one of the rational surfaces, the sheaves can be related to monopole or monopole strings in 
respectively four and five-dimensional supersymmetric gauge theory through geometric engineering [23,29]. If $S$ is a K3 surface, the sheaves correspond to (small) black holes in $\mathcal{N}=4$ supergravity also known as Dabholkar-Harvey states $[8,9]$. We specialize to the moduli space of sheaves with rank $r=1$ and 1 st and 2 nd Chern classes $c_{1} \in H^{2}(S, \mathbb{Z})$ and $c_{2} \in H^{4}(S, \mathbb{Z})$. These moduli spaces are isomorphic to the Hilbert scheme of $c_{2}$ points on $S$ (viewing $c_{2}$ as a number). Göttsche has determined the generating function of the Betti numbers of the Hilbert schemes [18]. We need to introduce some notation to explain his result.

Let $\mathcal{M}(n)$ be the Hilbert scheme of $n$ points. Let furthermore

$$
P(X, \zeta):=\sum_{i=0}^{2 \operatorname{dim}_{\mathbb{C}}(X)} \beta_{i}(X) \zeta^{i}
$$

be the Poincaré polynomial of $X$ with $\beta_{i}(X)$ the $i$ th Betti number of $X$. We choose the surface $S$ such that $\beta_{1}(S)=\beta_{3}(S)=0$. Then, we have [18]:

$$
\begin{aligned}
& \sum_{n \geq 0} \zeta^{-\frac{1}{2} \operatorname{dim}_{\mathbb{C}} \mathcal{M}(n)} P\left(\mathcal{M}(n), \zeta^{\frac{1}{2}}\right) q^{n} \\
& \quad=q^{\frac{\beta_{2}(S)+2}{24}}\left(\zeta^{\frac{1}{2}}-\zeta^{-\frac{1}{2}}\right) \frac{i}{\theta_{1}(w ; \tau) \eta(\tau)^{\beta_{2}(S)-1}}
\end{aligned}
$$

which precisely equals the function in (1.1) with $k=\beta_{2}(S)+2$. The coefficients $a_{m, k}(n)$ are in this context the Betti numbers of the moduli spaces. The expansion (1.2) in terms of $b_{J, k}(n)$ decomposes the cohomology in terms of $(2 J+1)$-dimensional $\mathrm{SL}(2)$ or $\mathrm{SU}(2)$ spin representations. For $J=0$ and $k=24$, our formula (1.3) confirms nicely the numerical estimates for $b_{0,24}(n)$ in [9, Appendix C]. Note that since this analysis is carried out in the socalled weak coupling or $D$-brane regime, and the coefficients $b_{J, k}(n)$ are not BPS indices, these coefficients cannot be claimed to count black holes with fixed angular momentum.

Equation (1.3) shows that in the context of this paper, the number of pure Higgs states has the same exponential growth as the total number of states, but that the number of pure Higgs states is smaller by a factor $n^{-\frac{3}{2}}$. Moreover, the number of $\mathrm{SU}(2)_{\text {spin }}$ multiplets increases with $J$ for small $J$. It is interesting to compare this with other known asymptotics of pure Higgs states. In particular, the authors $[4,11]$ considered this question for quiver moduli spaces in the limit of a large number of arrows between the nodes of a quiver with a potential. Bena et al. [4] demonstrated that the number of pure Higgs states for these quivers, $\beta_{\operatorname{dim}_{\mathbb{C}}(\mathcal{M})}(\mathcal{M})-\beta_{\operatorname{dim}_{\mathbb{C}}(\mathcal{M})+1}(\mathcal{M})$, is 
exponentially larger than the number of remaining SL(2) multiplets given by $\beta_{\operatorname{dim}_{\mathbb{C}}(\mathcal{M})+1}(\mathcal{M})$. We note that sheaves on toric surfaces relevant for this article also allow a description in terms of quivers [6,32]. The Hilbert scheme of $n \gg 1$ points corresponds to increasing dimensions of the spaces associated the nodes, with the number of arrows kept fixed. Thus we observe that the asymptotic behavior of the number of pure Higgs states in the two limits, large number of arrows or large dimensions, is rather different.

It will be interesting to understand better the significance of these different asymptotic behaviors. Moreover, we belief that application of the techniques in the present paper to partition functions for higher rank sheaves on surfaces $[26,27,35]$, and partition functions of black holes and quantum geometry $[5,16,22]$ will lead to to important novel insights.

The paper is organized as follows: In Section 2, we rewrite the functions of interest in terms of false theta functions and determine their Taylor expansion. Section 3 uses the Circle Method to prove our main theorem.

\section{Relation to false theta functions}

We start by writing the generating function of $a_{m, k}(n)$ for fixed $m$ and $k$ in terms of the functions $\vartheta_{m}(q)$ defined by:

$$
\vartheta_{m}(q):=\left(1+q^{|m|}\right) \sum_{n \geq 0}(-1)^{n} q^{\frac{n(n+1)}{2}+n|m|}-1 .
$$

Remark. We note that the property $\vartheta_{m}(q)=\vartheta_{-m}(q)$ of $\vartheta_{m}(q)$, continues to hold when $\vartheta_{m}(q)$ is defined with $|m|$ replaced by $m$.

The functions $\vartheta_{m}(q)$ are examples of false theta functions, which were first introduced by Rogers [31] and have attracted a lot of interest recently. Using the Rogers and Fine identity one can relate $\vartheta_{m}$ to so-called quantum modular forms, which are functions mimicking modular behavior on (subsets of) $\mathbb{Q}[15]$.

It is well known that the inverse theta function $\theta_{1}(w ; \tau)^{-1}$ can be written as a sum over its poles. See for more details, for example, $[2,17]$.

Proposition 2.1. We have for $|\zeta q|,\left|\zeta^{-1} q\right|<1$ :

$$
q^{\frac{k}{24}}\left(\zeta^{\frac{1}{2}}-\zeta^{-\frac{1}{2}}\right) h_{k}(w ; \tau)=\frac{1}{(q)_{\infty}^{k}} \sum_{m \in \mathbb{Z}} \vartheta_{m}(q) \zeta^{m}
$$

with $(q)_{\infty}:=\prod_{n=1}^{\infty}\left(1-q^{n}\right)$. 
Proof. The inverse theta function $\theta_{1}(w ; \tau)^{-1}$ is expressed as a sum over its poles by:

$$
\frac{i q^{\frac{1}{8}}\left(\zeta^{\frac{1}{2}}-\zeta^{-\frac{1}{2}}\right)}{\theta_{1}(w ; \tau)}=\frac{1}{(q)_{\infty}^{3}}(1-\zeta) \sum_{n \in \mathbb{Z}} \frac{(-1)^{n} q^{\frac{n(n+1)}{2}}}{1-\zeta q^{n}}
$$

Using geometric series expansion, we may rewrite (2.1) as

$$
\begin{gathered}
\frac{1}{(q)_{\infty}^{3}}+\frac{1}{(q)_{\infty}^{3}}(1-\zeta) \sum_{\substack{n>0 \\
m \geq 0}}(-1)^{n} q^{\frac{n(n+1)}{2}+n m} \zeta^{m} \\
+\frac{1}{(q)_{\infty}^{3}}\left(1-\zeta^{-1}\right) \sum_{\substack{n>0 \\
m \geq 0}}(-1)^{n} q^{\frac{n(n+1)}{2}+n m} \zeta^{-m}
\end{gathered}
$$

From this the statement of the proposition easily follows.

The function $\vartheta_{m}$ is not modular but may be nicely approximated by its Taylor expansion. For this we use the following general lemma (see [39] for the case of real functions).

Lemma 2.2. Let $f: \mathbb{C} \rightarrow \mathbb{C}$ be a $C^{\infty}$ function. Furthermore, we require that $f(x)$ and all its derivatives are of rapid decay for $\operatorname{Re}(x) \rightarrow \infty$. Then for $t \rightarrow 0$ with $\operatorname{Re}(t)>0$ and $a>0$, we have for any $N \in \mathbb{N}_{0}$ :

$$
\sum_{m=0}^{\infty} f((m+a) t)=\frac{1}{t} \int_{0}^{\infty} f(x) d x-\sum_{n=0}^{N} \frac{f^{(n)}(0)}{n !} \frac{B_{n+1}(a)}{n+1} t^{n}+O\left(t^{N+1}\right)
$$

Here $B_{n}(x)$ denotes the nth Bernoulli polynomial.

To use Lemma 2.2 we write for fixed $N \geq 1$ and $q=e^{-z}$

$$
q^{\frac{k}{24}} \vartheta_{m}(q)=: \sum_{\ell=1}^{N} d_{m, k}(\ell) z^{\ell}+O\left(z^{N+1}\right)
$$

Lemma 2.2 then gives 
Lemma 2.3. We have for $N \geq 1$

$$
\vartheta_{m}(q)=\left(1+q^{m}\right) q^{-\frac{1}{2}\left(m+\frac{1}{2}\right)^{2}} \sum_{\ell=0}^{N} c_{m}(\ell) z^{\ell}-1+O\left(z^{N+1}\right)
$$

with

$$
c_{m}(\ell):=\frac{(-1)^{\ell-1} 2^{\ell}}{\ell !(2 \ell+1)}\left(B_{2 \ell+1}\left(\frac{m}{2}+\frac{1}{4}\right)-B_{2 \ell+1}\left(\frac{m}{2}+\frac{3}{4}\right)\right) .
$$

In particular the first values for $d_{m, k}(\ell)$ are:

$$
\begin{aligned}
& d_{m, k}(1)=\frac{1}{4}, \quad d_{m, k}(2)=-\frac{k}{96}+\frac{1}{16}, \\
& d_{m, k}(3)=-\frac{m^{2}}{16}-\frac{k}{384}+\frac{k^{2}}{4608}+\frac{5}{192} .
\end{aligned}
$$

Proof. We may write $\vartheta_{m}(q)$ as:

$$
\begin{aligned}
\vartheta_{m}(q)= & \left(1+q^{m}\right) q^{-\frac{1}{2}\left(m+\frac{1}{2}\right)^{2}} \\
& \times \sum_{n \geq 0}\left(f\left(\left(n+\frac{m}{2}+\frac{1}{4}\right) \sqrt{z}\right)-f\left(\left(n+\frac{m}{2}+\frac{3}{4}\right) \sqrt{z}\right)\right)-1
\end{aligned}
$$

with $f(x):=e^{-2 x^{2}}$. Substitution of Lemma 2.2 gives the desired result.

Remark. We note that the case $m=0$ can be easily concluded from [38], where the asymptotics of the coefficients of $1 / 2\left(1-\vartheta_{0}(q)\right) /(q)_{\infty}^{k}$ for $k=1,2$ are determined.

\section{Use of the circle method}

In this section, we prove Theorem 1.1 following an approach by Wright [38]. To prove the theorem, we assume via symmetry that $m \geq 0$ and set

$$
F_{m, k}(q):=\sum_{n \geq 0} a_{m, k}(n) q^{n}
$$

By Proposition 2.1, we obtain that

$$
F_{m, k}(q)=\frac{1}{(q)_{\infty}^{k}} \vartheta_{m}(q)
$$


By Cauchy's Theorem, we have for $n \geq 1$

$$
a_{m, k}(n)=\frac{1}{2 \pi i} \int_{\mathcal{C}} \frac{F_{m, k}(q)}{q^{n+1}} d q
$$

where $\mathcal{C}$ is a circle surrounding 0 counterclockwise. We choose $e^{-\eta}$ for the radius of $\mathcal{C}$ with $\eta=\pi \sqrt{\frac{k}{6 n}}$ and split $\mathcal{C}$ into two $\operatorname{arcs} \mathcal{C}=\mathcal{C}_{1}+\mathcal{C}_{2}$, where $\mathcal{C}_{1}$ is the arc going counterclockwise from phase $-2 \eta$ to $2 \eta$ and $\mathcal{C}_{2}$ is its complement in $\mathcal{C}$. Consequently, we have

$$
a_{m, k}(n)=M+E
$$

with

$$
\begin{aligned}
M & :=\frac{1}{2 \pi i} \int_{\mathcal{C}_{1}} \frac{F_{m, k}(q)}{q^{n+1}} d q, \\
E & :=\frac{1}{2 \pi i} \int_{\mathcal{C}_{2}} \frac{F_{m, k}(q)}{q^{n+1}} d q .
\end{aligned}
$$

We will show that the main asymptotic contribution comes from $M$. Moreover we parametrize $q=e^{-z}$ with $\operatorname{Re}(z)=\eta$.

\subsection{The integral along $\mathcal{C}_{1}$}

In the integral along $\mathcal{C}_{1}$, we approximate $F_{m, k}$ by simpler functions. Firstly, recall that from the transformation law of the $\eta$-function [3, Theorem 3.1] we obtain:

$$
\frac{1}{\left(e^{-z} ; e^{-z}\right)_{\infty}}=\sqrt{\frac{z}{2 \pi}} e^{-\frac{z}{24}+\frac{\pi^{2}}{6 z}} \frac{1}{\left(e^{-\frac{4 \pi^{2}}{z}} ; e^{-\frac{4 \pi^{2}}{z}}\right)_{\infty}} .
$$

Thus we want to approximate $\frac{1}{(q)_{\infty}^{k}}$ by

$$
z^{\frac{k}{2}}(2 \pi)^{-\frac{k}{2}} e^{-\frac{k z}{24}} P_{k}\left(e^{-\frac{4 \pi^{2}}{z}}\right)
$$

where

$$
P_{k}(q):=\left(1+\sum_{\substack{24 \ell-k<0 \\ \ell>0}} p_{k}(\ell) q^{\ell}\right) q^{-\frac{k}{24}}
$$


To be more precise, we split

$$
M=M_{1}+E_{1}
$$

with

$$
\begin{gathered}
M_{1}:=\frac{1}{2 \pi i} \int_{\mathcal{C}_{1}} \frac{1}{q^{n+1}}\left(\frac{z}{2 \pi}\right)^{\frac{k}{2}} e^{-\frac{k z}{24}} P_{k}\left(e^{-\frac{4 \pi^{2}}{z}}\right) \vartheta_{m}(q) d q \\
E_{1}:=\frac{1}{2 \pi i} \int_{\mathcal{C}_{1}} \frac{1}{q^{n+1}} \vartheta_{m}(q)\left(\frac{1}{\left(e^{-z} ; e^{-z}\right)_{\infty}^{k}}-\left(\frac{z}{2 \pi}\right)^{\frac{k}{2}} e^{-\frac{k z}{24}} P_{k}\left(e^{-\frac{4 \pi^{2}}{z}}\right)\right) d q .
\end{gathered}
$$

We first bound $E_{1}$ which turns into the error term. Firstly, we obtain from (3.1)

$$
\frac{1}{\left(e^{-z} ; e^{-z}\right)_{\infty}^{k}}-\left(\frac{z}{2 \pi}\right)^{\frac{k}{2}} e^{-\frac{k z}{24}} P_{k}\left(e^{-\frac{4 \pi^{2}}{z}}\right)=O(1) .
$$

To bound $\vartheta_{m}$, we use that on $\mathcal{C}_{1}$

$$
|z|^{2}=\eta^{2}+\operatorname{Im}(z)^{2} \leq \eta^{2}+4 \eta^{2}
$$

Thus, by Lemma 2.3,

$$
\left|\vartheta_{m}(q)\right| \ll|z| \ll \eta,
$$

where throughout $g(x) \ll f(x)$ has the same meaning as $g(x)=O(f(x))$. Using that the length of $\mathcal{C}_{1}$ is $O(\eta)$, we may thus bound

$$
E_{1} \ll n^{-1} e^{\pi \frac{\sqrt{k n}}{\sqrt{6}}} .
$$

We next investigate $M_{1}$. We aim to approximate $\vartheta_{m}$ by its Taylor expansion given in Lemma 2.3 and thus we split

$$
M_{1}=M_{2}+E_{2}
$$

with

$$
\begin{gathered}
M_{2}:=\frac{1}{2 \pi i} \sum_{\ell=1}^{N} d_{m, k}(\ell) \int_{\mathcal{C}_{1}} \frac{1}{q^{n+1}}\left(\frac{z}{2 \pi}\right)^{\frac{k}{2}} P_{k}\left(e^{-\frac{4 \pi^{2}}{z}}\right) z^{\ell} d q \\
E_{2}:=\frac{1}{2 \pi i} \int_{\mathcal{C}_{1}} \frac{1}{q^{n+1}}\left(\frac{z}{2 \pi}\right)^{\frac{k}{2}} P_{k}\left(e^{-\frac{4 \pi^{2}}{z}}\right)\left(e^{-\frac{k z}{24}} \vartheta_{m}(q)-\sum_{\ell=1}^{N} d_{m, k}(\ell) z^{\ell}\right) d q .
\end{gathered}
$$


We first estimate $E_{2}$ and show that it contributes to the error term. By Lemma 2.3

$$
E_{2} \ll \int_{\mathcal{C}_{1}} e^{n \eta}|z|^{\frac{k}{2}+N+1} e^{\frac{\pi^{2} k}{6} \operatorname{Re}\left(\frac{1}{z}\right)} d z
$$

Since

$$
\frac{\operatorname{Re}(z)}{|z|^{2}} \leq \frac{1}{\operatorname{Re}(z)}=\frac{1}{\eta}
$$

we may bound

$$
n \eta+\frac{\pi^{2} k}{6} \operatorname{Re}\left(\frac{1}{z}\right) \leq \pi \sqrt{\frac{2 k n}{3}}
$$

Moreover, on $\mathcal{C}_{1}$

$$
|z|^{2}=x^{2}+y^{2} \leq \eta^{2}+4 \eta^{2} \ll \eta^{2} .
$$

As before, the path of integration may be estimated against $\eta$. Thus,

$$
\left|E_{2}\right| \ll \eta^{N+\frac{k}{2}+2} e^{\pi \sqrt{\frac{2 k n}{3}}} \ll n^{-\frac{N}{2}-\frac{k}{4}-1} e^{\pi \sqrt{\frac{2 k n}{3}}} .
$$

We next decompose

$$
M_{2}=(2 \pi)^{-\frac{k}{2}} \sum_{\ell=1}^{N} d_{m, k}(\ell) \sum_{24 j-k \leq 0} p_{k}(j) \mathcal{I}_{\ell+\frac{k}{2}, j}^{(k)},
$$

where for $s>0$

$$
\mathcal{I}_{s, j}^{(k)}:=\frac{1}{2 \pi i} \int_{\mathcal{C}_{1}} z^{s} e^{\frac{\pi^{2} k}{6 z}-\frac{4 \pi^{2} j}{z}+(n+1) z} d q
$$

These integrals may now be written in terms of the classical $I$-Bessel functions.

Lemma 3.1. We have

$$
\begin{aligned}
\mathcal{I}_{s, j}^{(k)=} & n^{\frac{-s-1}{2}}\left(\frac{\pi \sqrt{k-24 j}}{\sqrt{6}}\right)^{s+1} I_{-s-1}\left(\pi \sqrt{\frac{2}{3}(k-24 j) n}\right) \\
& +O\left(n^{-1-\frac{s}{2}} e^{\frac{\pi \sqrt{3 k n}}{2 \sqrt{2}}}\right) .
\end{aligned}
$$

Proof. Let $\mathcal{D}$ be the rectangular counterclockwise path from $-\infty-2 i \eta$ to $-\infty+2 i \eta$ with endpoints $\eta-2 i \eta$ and $\eta+2 i \eta$. Denote by $\mathcal{D}_{i}, i=1,2,3$, the paths (1) from $-\infty-2 i \eta$ to $\eta-2 i \eta$, (2) from $\eta-2 i \eta$ to $\eta+2 i \eta$ and (3) 
from $\eta+2 i \eta$ to $-\infty+2 i \eta$. Making the change of variables $q=e^{-z}$ gives that

$$
\mathcal{I}_{s, j}^{(k)}=\frac{1}{2 \pi i} \int_{\mathcal{D}_{2}} z^{s} e^{\frac{\pi^{2} k}{6 z}-\frac{4 \pi^{2} j}{z}+n z} d z .
$$

We next use the Residue Theorem to turn this integral into an integral over $\mathcal{D}$. For this, we bound the integrals along $\mathcal{D}_{1}$ and $\mathcal{D}_{3}$. We only give the details for $\mathcal{D}_{3}$. On this path, we may bound

$$
\left|\operatorname{Re}\left(\frac{1}{z}\right)\right| \leq \frac{1}{|z|} \leq \frac{1}{2 \eta}
$$

Writing $z=\eta(1+2 i)-u, 0 \leq u<\infty$ gives

$$
|z|=\sqrt{(\eta-u)^{2}+4 \eta^{2}} \ll \eta+u \text {. }
$$

Thus, the integral along $\mathcal{D}_{3}$ may be bounded by

$$
\begin{aligned}
& \ll e^{\frac{2 \pi^{2}}{\eta}\left(\frac{k}{24}-j\right)} \int_{0}^{\infty}(\eta+u)^{s} e^{n(\eta-u)} d u \\
& \ll e^{\frac{\pi^{2} k}{12 \eta}+n \eta}\left(\eta^{s} \int_{0}^{\eta} e^{-n u} d u+\int_{\eta}^{\infty} u^{s} e^{-n u} d u\right) .
\end{aligned}
$$

The second term is an incomplete Gamma function and thus exponentially small. Thus (3.3) may up to an exponentially small error be bounded by

$$
\eta^{s} e^{\frac{\pi^{2} k}{12 \eta}+n \eta} \frac{1}{n}\left(1-e^{-n \eta}\right) \ll n^{-1-\frac{s}{2}} e^{\frac{\pi \sqrt{3 k n}}{2 \sqrt{2}}} .
$$

In the remaining integral, we make the change of variables $z=\frac{t}{n}$ to get

$$
\mathcal{I}_{s, j}^{(k)}=n^{-s-1} \frac{1}{2 \pi i} \int_{\mathcal{D}} t^{s} e^{t+\frac{\pi^{2} k n}{6 t}-\frac{4 \pi^{2} j n}{t}} d t+O\left(n^{-1-\frac{s}{2}} e^{\frac{\pi \sqrt{3 k n}}{2 \sqrt{2}}}\right) .
$$

We now use the following representation of the $I$-Bessel function [1]

$$
I_{\ell}(2 \sqrt{z})=z^{\frac{\ell}{2}} \frac{1}{2 \pi i} \int_{-\infty}^{(0+)} t^{-\ell-1} \exp \left(t+\frac{z}{t}\right) d t
$$

where the integral is along any path looping from $-\infty$ around 0 back to $-\infty$ counterclockwise. Substitution into (3.4) gives the claim. 
Substitution of Lemma 3.1 into Equation (3.2) yields

$$
\begin{aligned}
M_{2}=(2 \pi)^{-\frac{k}{2}} & \sum_{\substack{1 \leq \ell \leq N \\
24 j-k \leq 0}} d_{m, k}(\ell) p_{k}(j) n^{-\frac{2+2 \ell+k}{4}}\left(\frac{\pi \sqrt{k-24 j}}{\sqrt{6}}\right)^{1+\ell+\frac{k}{2}} \\
& \times I_{-1-\ell-\frac{k}{2}}\left(\pi \sqrt{\frac{2}{3}(k-24 j) n}\right)+O\left(n^{-\frac{3}{2}-\frac{k}{4}} e^{\frac{\pi \sqrt{3 k n}}{2 \sqrt{2}}}\right) .
\end{aligned}
$$

\subsection{The integral along $\mathcal{C}_{2}$}

On $\mathcal{C}_{2}, \operatorname{Im}(z)$ varies from $-2 \eta$ to $-2 \pi+2 \eta$. Using a rough bound for the theta function, we find

$$
\left|\vartheta_{m}(q)\right| \ll 2 \sum_{n \geq 1} e^{-\frac{n}{2}(n+1+2 m) \operatorname{Re}(z)}+1 \ll 2 \sum_{n \geq 0} e^{-n \operatorname{Re}(z)}=\frac{2}{1-e^{-\eta}} \ll \frac{1}{\eta}
$$

Using (3.1) we obtain the bound

$$
\frac{1}{\left(e^{-z} ; e^{-z}\right)_{\infty}} \ll e^{\frac{\pi^{2}}{6} \operatorname{Re}\left(\frac{1}{z}\right)}
$$

Now

$$
\operatorname{Re}\left(\frac{1}{z}\right)=\frac{\eta}{\eta^{2}+\operatorname{Im}(z)^{2}} \leq \frac{1}{5 \eta} .
$$

Thus (3.5) may be estimated against $\exp \left(\frac{\pi^{2}}{30 \eta}\right)$. This gives that:

$$
E \ll e^{\frac{\pi^{2} k}{30 \eta}+n \eta} \ll e^{\frac{\pi}{5} \sqrt{6 n k}}
$$

This is exponentially smaller than the other errors. Combining this with the results of Section 3.1 therefore gives Theorem 1.1.

\section{Acknowledgments}

The research of the authors was supported by the Alfried Krupp Prize for Young University Teachers of the Krupp foundation. After this paper was submitted to the arXiv, we learned from Paul de Lange that for special cases the main terms were also obtained by [7]. We are grateful to him for the correspondence. We also thank Roland Mainka, Boris Pioline, Rob Rhoades and Miguel Zapata Rolón for useful correspondence. 


\section{References}

[1] M. Abramovitz and I. Stegun, Handbook of Mathematical Functions with Formulas, Graphs, and Mathematical Tables, Dover Publications, New York, 1972.

[2] G. Andrews and F. Garvan, Dyson's crank of a partition, Bull. Amer. Math. Soc. 18 (1988), 161-171.

[3] T. Apostol, Modular Functions and Dirichlet Series in Number Theory, Springer-Verlag, New York-Heidelberg, 1976.

[4] I. Bena, M. Berkooz, J. de Boer, S. El-Showk and D. van den Bleeken, Scaling BPS Solutions and pure-Higgs States, J. High Energy Phys. 11 (2012) 171; arXiv:1205.5023 [hep-th].

[5] J. de Boer, M. Cheng, R. Dijkgraaf, J. Manschot and E. Verlinde, A Farey Tail for Attractor Black Holes, J. High Energy Phys. 11 (2006) 024; arXiv:hep-th/0608059.

[6] A. Bondal, Representation of associative algebras and coherent sheaves, Math. USSR Izvestiya 34 (1990) 23-42.

[7] T.L. Curtright and C.B. Thorn, symmetry patterns in the mass spectra of dual string models, Nucl. Phys. B 274 (1986) 520.

[8] A. Dabholkar and J.A. Harvey, Nonrenormalization of the superstring tension, Phys. Rev. Lett. 63 (1989) 478.

[9] A. Dabholkar, F. Denef, G.W. Moore and B. Pioline, Precision counting of small black holes, J. High Energy Phys. 10 (2005) 096; arXiv:hep-th/0507014.

[10] A. Dabholkar, S. Murthy and D. Zagier, Quantum black holes, wall crossing, and mock modular forms, arXiv:1208.4074 [hep-th].

[11] F. Denef and G. Moore, Split states, entropy enigmas, holes and halos, J. High Energy Phys. 11 (2011) 129; arXiv:hep-th/0702146 [hep-th].

[12] P. Di Francesco, P. Mathieu and D. Senechal, Conformal field theory, Springer, New York, USA, 1997.

[13] R. Dijkgraaf, G. Moore, E. Verlinde and H. Verlinde, Elliptic genera of symmetric products and second quantized strings, Commun. Math. Phys. 185 (1997) 197-209; arXiv: hep-th/9608096. 
[14] M. Eichler and D. Zagier, The Theory of Jacobi Forms. Birkhäuser, Boston, 1985.

[15] A. Folsom, K. Ono and R. Rhoades, q-series and quantum modular forms, Forum Math. Pi 1 (2013).

[16] D. Gaiotto, A. Strominger and X. Yin, The M5-brane elliptic genus: modularity and BPS states, J. High Energy Phys. 08 (2007) 070; arXiv:hep-th/0607010.

[17] F.G. Garvan, New combinatorial interpretations of Ramanujan's partition congruences mod 5,7 and 11, Trans. Amer. Math. Soc. 305 (1988) 47-77.

[18] L. Göttsche, The Betti numbers of the Hilbert scheme of points on a smooth projective surface, Math. Ann. 286 (1990), 193-207.

[19] L. Göttsche and D. Zagier, Jacobi forms and the structure of Donaldson invariants for 4-manifolds with $b_{+}=1$, Selecta Math. New Ser. 4 (1998) 69-115; arXiv:alg-geom/9612020.

[20] P. Hammond and R. Lewis, Congruences in ordered pairs of partitions, Int. J. Math. Sci. 47 (2004), 2509-2512.

[21] G. Hardy and S. Ramanujan, Asymptotic formulae in combinatory analysis, Collected papers of Srinivasa Ramanujan, 244, AMS Chelsea Publication, Providence, RI, 2000.

[22] M.-x. Huang, A. Klemm, M. Marino and A. Tavanfar, Black holes and large order quantum geometry, Phys. Rev. D 79 (2009) 066001; arXiv:0704.2440 [hep-th].

[23] S. Katz, A. Klemm and C. Vafa, Geometric engineering of quantum field theories, Nucl. Phys. B 497 (1997) 173-195; arXiv:hep-th/9609239.

[24] T. Kawai, Y. Yamada and S. Yang, Elliptic genera and $N=2$ superconformal field theory, Nucl. Phys. B 414 (1994) 191-212; arXiv:hep-th/9306096.

[25] S. Lee, Z. Wang and P. Yi, Quiver invariants from intrinsic Higgs states, J. High Energy Phys. 07 (2012), 169; arXiv:1205.6511 [hep-th].

[26] J. Manschot, BPS invariants of $N=4$ gauge theory on a surface, Commun. Num. Theor. Phys. 6 (2012), 497-516; arXiv:1103.0012 [math-ph]. 
[27] J. Manschot, BPS invariants of semi-stable sheaves on rational surfaces, Lett. Math. Phys. 103 (2013) 895-918.

[28] J. Manschot, B. Pioline and A. Sen, From black holes to quivers, J. High Energy Phys. 11 (2012), 023; arXiv:1207.2230 [hep-th].

[29] D. Morrison and N. Seiberg, Extremal transitions and five-dimensional supersymmetric field theories, Nucl. Phys. B 483 (1997), 229-247; arXiv:hep-th/9609070.

[30] H. Rademacher and H. Zuckerman, On the fourier coefficients of certain modular forms of positive dimension, Ann. Math. 39(2) (1938), 433-462.

[31] L. Rogers, On two theorems of combinatory analysis and some allied identities, Proc. Lond. Math. Soci. (2), 16 (1917), 315-336.

[32] A.N. Rudakov, Helices and vector bundles: seminaire Rudakov, Cambridge University Press, Cambridge, 1990.

[33] J.G. Russo and L. Susskind, Asymptotic level density in heterotic string theory and rotating black holes, Nucl. Phys. B 437 (1995), 611; arXiv: hep-th/9405117.

[34] A. Sen, Arithmetic of Quantum entropy function, J. High Energy Phys. 08 (2009), 068; arXiv:0903.1477 [hep-th].

[35] K. Yoshioka, The Betti numbers of the moduli space of stable sheaves of rank 2 on $\mathbb{P}^{2}$, J. Reine. Angew. Math. 453 (1994), 193-220.

[36] E. Witten, Phase transitions in $M$ theory and $F$ theory, Nucl. Phys. B 471 (1996), 195-216; arXiv:hep-th/9603150.

[37] E. Wright, Stacks, Q. J. Math. 19 (1968), 313-320.

[38] E. Wright, Stacks (II), Q. J. Math. 22 (1971), 107-116.

[39] D. Zagier, The Mellin transform and other useful analytic techniques, Appendix to E. Zeidler, Quantum Field Theory I: Basics in Mathematics and Physics. A Bridge Between Mathematicians and Physicists, Springer-Verlag, Berlin, 2006 305-323.

[40] D. Zagier, Quantum modular forms, In Quanta of Maths: Conference in honour of Alain Connes, Clay Mathematics Proceedings 11, AMS and Clay Mathematics Institute 2010, 659-675. 
Mathematical Institute

UNIVERSITY OF COLOGNE

WEYERTAL 86-90

50931 COLOGNE

GERMANY

E-mail address: kbringma@math.uni-koeln.de

Camille Jordan Institute

UNIVERSITY OF LYON

43 BOULEVARD DU 11 NOVEMBRE 1918

69622 VilleurbanNe CEDEX

FRANCE

E-mail address:jan.manschot@univ-lyon1.fr

Received May 15, 2013 
